\title{
Gastro-oesophageal reflux and gastric aspiration in lung transplant patients with or without chronic rejection
}

\author{
K. Blondeau*, V. Mertens*, B.A. Vanaudenaerde ${ }^{\#}$, G.M. Verleden\#, D.E. Van \\ Raemdonck*, D. Sifrim* and L.J. Dupont ${ }^{\#}$
}

ABSTRACT: Acid gastro-oesophageal reflux (GOR) and gastric aspiration have been labelled as risk factors for chronic rejection bronchiolitis obliterans syndrome (BOS) after lung transplantation (LTX). The present study aimed to further characterise GOR (both acid and nonacid) and the degree of gastric aspiration in LTx recipients both with and without BOS.

Impedance-pH recordings were used for GOR detection. Pepsin and bile acid levels were measured in bronchoalveolar lavage fluid (BALF).

A total of $48 \%$ of patients had increased GOR, of which $27 \%$ had exclusively increased nonacid reflux. Cystic fibrosis patients had the highest prevalence of GOR. Pepsin was found in BALF of all patients and bile acids in BALF of $50 \%$ of the patients. Patients with BOS had neither increased GOR nor elevated pepsin in BALF. However, $70 \%$ of the patients with BOS had bile in BALF compared with $31 \%$ of stable patients. Proton pump inhibitor (PPI) treatment reduced acid reflux but did not affect nonacid reflux. Moreover, pepsin and bile levels in BALF were not reduced by PPI.

One-half of the lung transplant patients had increased reflux, and nonacid reflux was common. Gastric aspiration occurred in most lung transplant patients. Pepsin was a more general marker and bile acids a more specific marker that might be associated with bronchiolitis obliterans syndrome. Proton pump inhibitor treatment did not prevent nonacid reflux and gastric aspiration.

KEYWORDS: Bronchiolitis obliterans syndrome, gastro-oesophageal reflux, transplantation

ung transplantation (LTx) has now become an effective therapeutic option for the treatment of different end-stage pulmonary disorders, improving the quality of life and extending survival [1]. A significant contributor to the limited long-term survival is the development of chronic allograft rejection [1]. Bronchiolitis obliterans syndrome (BOS), identified as a persistent drop in the forced expiratory volume in one second (FEV1) after transplantation, is the clinical correlate of chronic rejection and is caused by obliteration of the terminal bronchioles [2]. The pathophysiology of BOS is poorly understood, but both immunological and nonimmunological mechanisms are involved [3-5].

Gastro-oesophageal reflux (GOR) has been implicated as a potential non-alloimmune cause of BOS. Standard oesophageal pH-metry indicated an increased oesophageal acid exposure in $70 \%$ of LTx patients [6, 7]. Luminal gastric components, such as pepsin and bile acids, have been demonstrated in bronchial material of LTx recipients [8, 9]. D'OvIDIO et al. [10] demonstrated increased bile acids levels in bronchoalveolar lavage fluid (BALF) in $22 \%$ of LTx patients, which were associated with reduced freedom from BOS [10]. Anti-reflux fundoplication surgery has been associated with improved allograft function [11-13]. These data suggest a possible causal link between GOR and the development of BOS $[7,12]$.

Although GOR and gastric aspiration seem to occur in LTx recipients, understanding their role in the development of BOS requires additional information. Furthermore, it is necessary to establish the patient profile, on the basis of reflux indices and/or markers of gastric aspiration that might benefit from anti-reflux intervention.

So far, only acid GOR $(\mathrm{pH}<4)$ has been measured in LTx patients. However, less acidic reflux $(\mathrm{pH}$ >4) might also induce aspiration of harmful gastric components. Impedance-pH monitoring is currently considered the most sensitive method for GOR detection and allows the recognition of
AFFILIATIONS

*Centre for Gastroenterological Research, and

\#Lung Transplant Unit, University Hospital Gasthuisberg, Katholieke Universiteit, Leuven, Belgium.

CORRESPONDENCE

L.J. Dupont

Division of Respiratory Medicine University Hospital Gasthuisberg 49 Herestraat

B-3000 Leuven Belgium

Fax: 3216346803

E-mail: lieven.dupont@

uz.kuleuven.be

Received:

May 302007

Accepted after revision:

November 222007

SUPPORT STATEMENT

This work was supported by a grant from the Fonds voor

Wetenschappelijk Onderzoek (FWO)

Vlaanderen and a grant from the "Geconcerteerde Onderzoeksactie" of the Katholieke Universiteit Leuven. D. Sifrim received a research grant from Sandhill Scientific (Denver, CO USA). L.J. Dupont is a part-time senior research fellow of the Fonds Wetenschappelijk Onderzoek Vlaanderen.

STATEMENT OF INTEREST A statement of interest for this study can be found at www.erj.ersjournals.com/misc/ statements.shtml 
acid and nonacid reflux [14]. Gastric aspiration can be analysed by measuring pepsin or bile acids levels in BALF. A significant proportion of LTx patients are empirically treated with proton pump inhibitors (PPI). It is unknown whether this affects the prevalence of reflux and risk of gastric aspiration.

The primary aim of the present study was to characterise the prevalence and type of GOR (acid and nonacid) and the degree of gastric aspiration in LTx recipients both with and without BOS. The secondary aim was to assess the prevalence and degree of acid and nonacid GOR and gastric aspiration in a separate group of LTx patients, studied while being treated with PPI.

\section{METHODS}

\section{Design and patient recruitment}

The present study was performed as a cross-sectional study, recruiting patients unselectively from the LTx recipients (LTx between January 1996 and June 2005). Only patients with a survival and follow-up $>1$ yr were included. For each patient, FEV1 (L; \% predicted and \% personal best) was available at the day of the impedance-pH study. BOS, defined as an irreversible decline in FEV1, was graded according to International Society for Heart and Lung Transplantation criteria [15]. Acute rejection was excluded both clinically and by means of transbronchial biopsies. Infection was excluded on the basis of clinical presentation and sputum or BALF microbiology. Recipients with anastomotic complications or incomplete pulmonary function test data were excluded.

All patients received standard immunosuppressive therapy comprising cyclosporine or tracrolimus, azathioprine or mycophenolate and methylprednisolone.

Although one of the aims was to assess the impact of treatment with PPI on the prevalence of GOR, nearly all patients refused to undergo two separate $\mathrm{pH}$-impedance recordings (one while "off" PPI and one while "on" PPI). As a result, patients were recruited in two different study cohorts. The first and largest study cohort consisted of patients evaluated with $\mathrm{pH}$ impedance either without or after discontinuing acid suppression treatment or pro-kinetic drugs for $\geqslant 2$ weeks. In the first study cohort, the prevalence of GOR and aspiration was compared between patients with and without BOS. The second study cohort, consisting of a smaller number of patients, was evaluated with $\mathrm{pH}$ impedance while being treated with omeprazole $20 \mathrm{mg}$ b.i.d.

\section{Impedance-pH monitoring}

GOR was assessed at the moment of inclusion using ambulatory 24-h oesophageal impedance-pH monitoring. The impedance-pH recording was independently analysed for GOR using criteria described in a recent consensus report [16]. Several reflux indices were measured: acid exposure, number of acid and nonacid reflux events, volume exposure and proximal extent of reflux. Detailed methodology can be found in the Appendix.

\section{Detection of gastric aspiration (pepsin and bile acids in BALF)}

For every patient that participated in the present study, a BAL sample was obtained during the next scheduled routine bronchoscopy following the $\mathrm{pH}$-impedance recording. BALF was performed by wedging the bronchoscope into a subsegmental bronchus of the right middle lobe or lingula, then instilling two aliquots of saline and subsequently recovering the fluid by gentle manual suction. Pepsin and bile acids were determined in BALF. In the patients of the first study cohort, acid suppression and pro-kinetic treatment was stopped before the bronchoscopy while the patients in the second study cohort continued treatment with PPI.

Fourteen BALF samples of "nontransplant" subjects requiring a bronchoscopy (11 lung cancer, two chronic obstructive pulmonary disease, one lymphoma) were collected for comparative analysis. BALF was performed and analysed identically.

\section{Pepsin detection}

Pepsin was measured using an ELISA. Samples were incubated with a primary polyclonal antibody to porcine pepsine $(1: 5,000)$ and a secondary antibody (goat immunoglobulin G) labelled with horseradish peroxidase $(1: 10,000)$. Tetramethylbenzidine was added and colour change was measured using a spectrophotometer. The specificity of the assay was verified using Western blot analysis and the lowest level of accurate detection was $1 \mathrm{ng} \cdot \mathrm{L}^{-1}$.

\section{Bile acids}

Bile acid determination was performed using a commercially available enzymatic assay (Bioquant, San Diego, CA, USA). The lowest level of accurate detection allowed by this technique was $0.2 \mu \mathrm{mol} \cdot \mathrm{L}^{-1}$.

\section{Statistical analysis}

Deviations from Gaussian distribution were tested using the Kolmogorov-Smirnov test. Comparisons between groups were carried out using one-way ANOVA and subsequent nonparametric testing. Categorical data were analysed using Fischer's exact test. Correlations were made using Pearson's test or Spearman's test, as appropriate. All results are expressed as median (25th-75th percentile), unless otherwise stated.

\section{RESULTS}

The prevalence of GOR and gastric aspiration, as well as the comparison between BOS and no BOS patients, was analysed in the first study cohort. The results in the second study cohort are listed in a separate paragraph.

\section{Patient characteristics}

The first study cohort (off PPI patients) consisted of 45 LTx recipients (29 males, median (range) age 52 (19-69) yrs). The second study cohort (on PPI patients) consisted of a separate group of 18 patients (eight males, age 58 (22-66) yrs). All 18 patients had adequate control of the gastric acid secretion $(<50 \%$ of the recording time with gastric $\mathrm{pH}<4)$. The underlying diagnoses and patient characteristics are listed according to BOS stage in table 1 . The mean time between the LTx and reflux assessment with impedance-pH monitoring was $36(14-45)$ months.

\section{GOR in patients studied off PPI}

Typical reflux symptoms (heartburn or regurgitation) were present in 18 out of 45 patients (11 patients prior to LTx, seven 


\section{TABLE 1 Patient characteristics}

\begin{tabular}{|c|c|c|c|c|}
\hline & \multicolumn{3}{|c|}{ Study cohort 1 (off PPI) } & \multirow{2}{*}{$\begin{array}{l}\text { Study cohort } 2 \text { (on PPI) } \\
\text { BOS } 0^{+} \text {BOS } 0 p^{\S} \text { BOS } \geqslant 1^{f}\end{array}$} \\
\hline & Bos 0 & Bos op & BOS $\geqslant 1$ & \\
\hline Subjects & 25 & 9 & 11 & \\
\hline Male/female & $19 / 6$ & $3 / 6$ & $7 / 4$ & $10 / 8$ \\
\hline Age yrs & $51 \pm 11$ & $53 \pm 11$ & $46 \pm 13$ & $53 \pm 13$ \\
\hline CF/other $\# \#$ & $4 / 21$ & $2 / 7$ & $1 / 10$ & $2 / 16$ \\
\hline SSLTx/SLTX/HLTX & $19 / 5 / 1$ & $3 / 5 / 1$ & $8 / 1 / 2$ & $11 / 6 / 1$ \\
\hline Weeks after LTx & $114 \pm 69$ & $130 \pm 54$ & $227 \pm 124$ & $112 \pm 60$ \\
\hline Patients with a history of $A R \geqslant 2$ episodes & 4 & 2 & 5 & 4 \\
\hline Patients colonised at study day & 4 & 1 & 3 & 4 \\
\hline CMV donor/receptor $1 / 2 / 3 \%$ & $7 / 9 / 9$ & $5 / 2 / 2$ & $4 / 3 / 4$ & $13 / 2 / 3$ \\
\hline
\end{tabular}

Data are presented as $n$ or mean \pm SD. PPI: proton pump inhibitor; BOS: bronchiolitis obliterans syndrome; BOS 0: no BOS; BOS Op: probable BOS; BOS $\geqslant 1$ : BOS grade 1 or higher; CF: cystic fibrosis; SSLTx: double lung transplantation; SLTX: single lung transplantation; HLTx: heart-lung transplantation; AR: acute rejection; CMV:

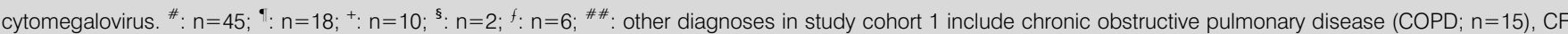
$(n=7)$, pulmonary fibrosis $(n=6)$, primary pulmonary hypertension $(n=5)$, bronchiectasis $(n=3)$, lymphangioleiomyomatosis $(L A M ; n=1)$, sarcoidosis $(n=1)$, pulmonary fibrosis due to congenital dyskeratosis $(n=1)$, hypersensitivity pneumonitis $(n=2), \alpha_{1}$-antitrypsin deficiency emphysema $(n=2)$ and Williams-Campbell syndrome ( $\left.n=1\right)$ Other diagnoses in study cohort 2 include COPD $(n=8), \operatorname{CF}(n=2)$, pulmonary fibrosis $(n=3)$, primary pulmonary hypertension $(n=1)$, bronchiectasis $(n=1)$, LAM $(n=1)$, obliterative bronchiolitis (induced by a viral infection during childhood; $n=1$ ) and histiocytosis $X(n=1)$. " $"$ : CMV status of the $L T x$ donor (D) and receptor $(R)$ are as follows: 1) $D+/ R+$ or $D-/ R+$, 2) $D-/ R-$, and 3) $D+/ R-$.

after LTx). A total of $22(49 \%)$ patients had increased GOR defined as having one or more abnormal reflux indices (increased acid exposure, volume exposure or number of reflux events). Increased acid reflux was noted in 16 (73\%), whereas six patients $(27 \%)$ had only increased nonacid reflux. Median values of different reflux are displayed in table 2.

Patients with cystic fibrosis (CF) had significantly more reflux episodes compared with patients with other diagnoses (fig. 1). The oesophageal acid exposure and the proximal extent of reflux were also significantly increased in patients with $\mathrm{CF}$ compared with other LTx patients (8.3 (3.4-38.15)\% versus 2.05 $(1.0-5.05) \%, \mathrm{p}=0.001$; and $19(11-61) \%$ versus $7 \quad(3-16) \%$, $p=0.005$, respectively). Other reflux indices were unaffected by the type of end-stage lung disease. The type of transplant surgery had no effect on any of the reflux parameters.

TABLE 2 Gastro-oesophageal reflux indices

\begin{tabular}{lcc} 
& $\begin{array}{c}\text { Study } \\
\text { population }\end{array}$ & $\begin{array}{c}\text { Patients studied } \\
\text { on PPI }\end{array}$ \\
\hline Subjects $\mathbf{n}$ & 45 & 18 \\
Oesophageal acid exposure \% & $3.1(1.0-6.4)$ & $0.5(0.1-2.3)$ \\
Oesophageal volume exposure \% & $0.7(0.3-1.4)$ & $0.75(0.4-1.4)$ \\
Total number of reflux events $\mathbf{2 4} \mathbf{~}^{\mathbf{- 1}}$ & $33(17-49)$ & $34(18-52)$ \\
Acid reflux events $\mathbf{n}$ & $16(7-26)$ & $2(0-8)$ \\
Nonacid reflux events $\mathbf{n}$ & $15(6-20)$ & $26(18-45)$ \\
Proximal extent of reflux at $\mathbf{1 5} \mathbf{c m}^{\#}$ & $9(3-19)$ & $13(2-17)$ \\
\hline
\end{tabular}

Data are presented as median (25th-75th percentile), unless otherwise indicated. PPI: proton pump inhibitor. ${ }^{*}$ : i.e. reflux events $>15 \mathrm{~cm}$.

\section{Gastric aspiration in patients studied off PPI}

All LTx patients had detectable levels of pepsin in their BALF. The median pepsin concentration detected in the BALF of LTx recipients was significantly higher than in nontransplant patients (541 (187-946) versus $24(0-25) \mathrm{ng} \cdot \mathrm{mL}^{-1}$, respectively, $\mathrm{p}<0.0001$; fig. 2). The pepsin concentration was slightly higher in CF patients when compared with other LTx patients, but this was not significant $(769(566-1,190)$ versus 488 (166918) $\mathrm{ng} \cdot \mathrm{mL}^{-1}$, respectively; $\mathrm{p}=$ nonsignificant). The BALF pepsin concentration was similar in patients who underwent double LTx (SSLTx; $\left.601(285-1,001) \mathrm{ng} \cdot \mathrm{mL}^{-1}\right)$, left single lung

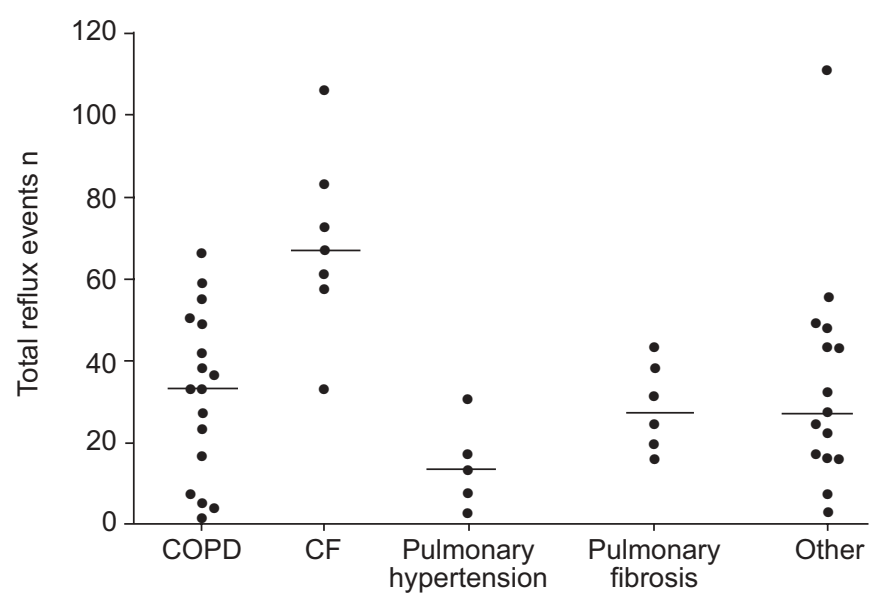

FIGURE 1. The total number of reflux events in patients with different underlying diagnosis. Patients with cystic fibrosis ( $C F ; n=67$ ) had significantly $(p=0.0052)$ more reflux episodes compared with patients with chronic obstructive pulmonary disease (COPD; $32(19-44))$, pulmonary fibrosis $(n=38)$, pulmonary hypertension $(n=19)$ and patients with other diagnosis (23 (9-43)). 


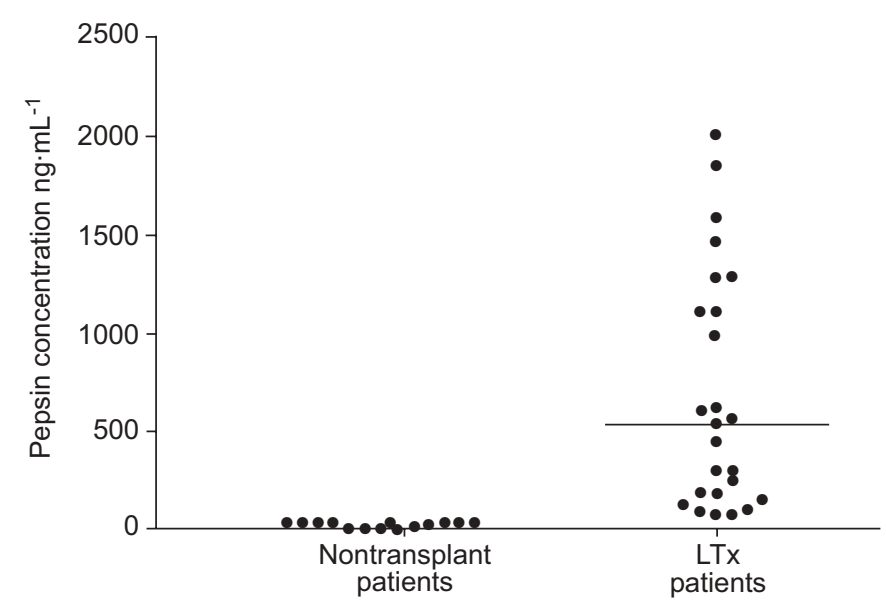

FIGURE 2. The concentration of pepsin in the bronchoalveolar lavage fluid was significantly higher in lung transplant (LTX) recipients (532 (1841,190) $\left.\mathrm{ng} \cdot \mathrm{mL}^{-1}\right)$ compared with controls $\left(23.83(0-25) \mathrm{ng} \cdot \mathrm{mL}^{-1}\right)$.

transplantation (SLTx; $\left.315(118-715) \mathrm{ng} \cdot \mathrm{mL}^{-1}\right)$, right SLTx (367 $\left.(112-1517) \mathrm{ng} \cdot \mathrm{mL}^{-1}\right)$ and heart-lung transplantation (HLTx; 93 $\left.(59-549) \mathrm{ng} \cdot \mathrm{mL}^{-1}\right)$. Pepsin levels were significantly correlated with the amount of neutrophils found in BALF $(r=0.3692$, $\mathrm{p}=0.014)$.

A total of 22 patients (49\%) had detectable bile acids in BALF as opposed to none of the nontransplant controls. Six out of the seven CF patients (86\%) had detectable bile acids in BALF. This was significantly higher when compared with non-CF patients (16 out of $38 ; \mathrm{p}=0.04$ ). The proportion of patients with bile acids in BALF was similar in patients who received SSLTx (15 out of 30), SLTx (five out of 11) or HLTx (two out of four).

\section{GOR and BOS in patients studied off PPI}

All of the 24-h reflux parameters (acid exposure, bolus exposure, number of reflux events and proximal extent) were similar in patients with $\mathrm{BOS}$ grade $\geqslant 1(\mathrm{BOS} \geqslant 1)$, patients with probable BOS (BOS 0p) and stable patients (BOS 0; table 3).

The number of patients having one or more abnormal reflux indices was not significantly different in the subgroups with different BOS stages (14 out of 25 BOS 0 patients; two out of nine BOS $0 p$ patients and six out of 11 BOS $\geqslant 1$ patients; $\mathrm{p}=$ nonsignificant).

\section{Gastric aspiration and BOS in patients studied off PPI}

Pepsin levels in BALF were similar in patients with BOS $\geqslant 1$, patients with BOS 0p and stable patients (BOS 0; 492 (181-987), 435 (171-795) and $728(184-1,282) \mathrm{ng} \cdot \mathrm{mL}^{-1}$, respectively; $\mathrm{p}=$ nonsignificant).

There was no significant correlation between pepsin in BALF and FEV1, regardless of whether the FEV1 was expressed as \% pred, \% personal best or in absolute volume.

The median level of bile acids in BALF was slightly higher in patients with BOS $\left(0.5(0-0.8) \mu \mathrm{mol} \cdot \mathrm{L}^{-1}\right)$ compared with patients with BOS $0 \mathrm{p}\left(0.2(0-1.2) \mu \mathrm{mol} \cdot \mathrm{L}^{-1}\right)$ and patients with BOS $0\left(0.1(0-0.32) \mu \mathrm{mol} \cdot \mathrm{L}^{-1}\right)$, although this did not reach statistical significance $(\mathrm{p}=0.1)$. However, significantly more patients with BOS (12 out of 17) had detectable bile in the BALF compared with stable patients (five out of $16 ; \mathrm{p}=0.03$; fig. 3).

There was no significant correlation between the concentration of bile acids in BALF and the FEV1.

\section{GOR and gastric aspiration in patients studied on PPI}

Seven out of 18 patients on PPI had increased GOR, of which five out of seven patients (71\%) had increased weakly acidic reflux (number of episodes). Two patients had increased oesophageal acid exposure, despite adequate control of gastric acid secretion.

The oesophageal acid exposure and the number of acid reflux events was significantly reduced compared with the patients studied off PPI $(0.5(0.1-2.3)$ versus $3.1(1.0-6.4) \% ; \mathrm{p}=0.001$, and $2(0-8)$ versus $16(7-26) \% ; \mathrm{p}=0.002$, respectively). PPI treatment was not associated with reduced total number of reflux events, reduced number of weakly acidic reflux, reduced volume exposure or reduced proximal extent of reflux (table 2).

All 18 patients had detectable pepsin in BALF. Pepsin levels in patients on PPI $\left(658(146-1,044) \mathrm{ng} \cdot \mathrm{mL}^{-1}\right)$ were similar to pepsin levels in patients studied off PPI (541 (187-946) $\left.\mathrm{ng} \cdot \mathrm{mL}^{-1}\right)$. Nine out of 18 patients had bile in BALF. The proportion of patients on PPI (nine out of 18) with bile in BALF was similar compared with patients studied off PPI (22 out of 45).

TABLE 3 Gastro-oesophagal reflux indices in patients with and without bronchiolitis obliterans syndrome (BOS)

\begin{tabular}{|c|c|c|c|}
\hline Subjects $n$ & 27 & 9 & 1 \\
\hline Oesophageal volume exposure \% & $0.8(0.54-1.7)$ & $0.4(0.3-1.5)$ & $0.5(1.2-1.3)$ \\
\hline Total number of reflux events $24 h^{-1}$ & $33(29-48)$ & $49(36-71)$ & $32(9-45)$ \\
\hline Acid reflux events $n$ & $17(13-24)$ & $4(0-10)$ & $6(2-20)$ \\
\hline Nocturnal reflux events $n$ & $4(2-6)$ & $2(1-6)$ & $5(3-9)$ \\
\hline
\end{tabular}

Data are presented as median (25th-75th percentile), unless otherwise indicated. BOS 0: BOS not present; BOS Op: probable BOS; BOS 1-2-3: BOS grade 1, 2 or 3 present. ${ }^{\#}$ : i.e. reflux events $>15 \mathrm{~cm}$. 


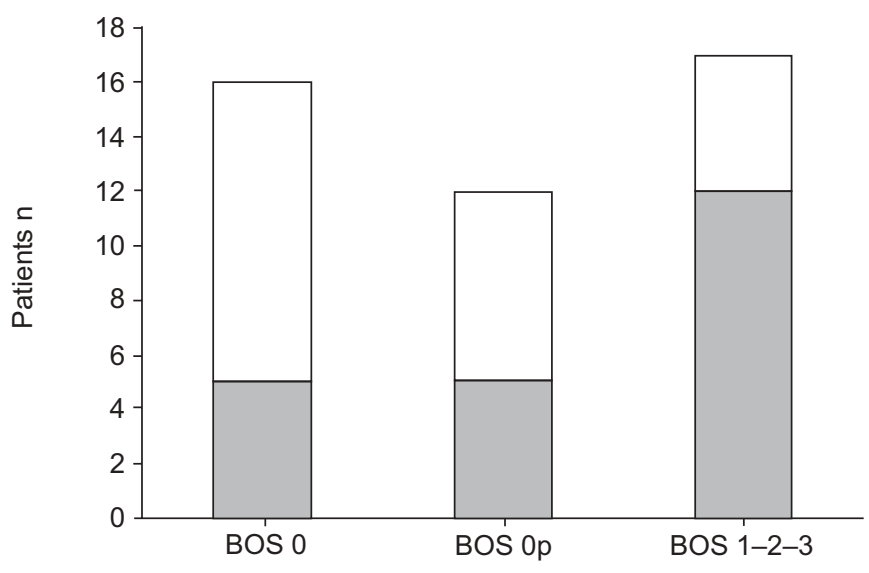

FIGURE 3. The proportion of patients with bile acids in the bronchoalveolar lavage fluid (BALF; $\square$ ) compared with no bile $(\square)$ in relation to the bronchiolitis obliterans syndrome (BOS) stage. Significantly more patients with BOS had detectable bile in the BALF compared with stable patients $(p=0.03)$. BOS 0 : BOS not present; BOS Op: probable BOS; BOS 1-2-3: BOS grade 1, 2 or 3 present.

\section{DISCUSSION}

It has been suggested that non-alloimmune factors, such as infections and GOR, contribute to the development of BOS after LTx $[5,15,18]$. In the current cross-sectional study, the presence of reflux and gastric aspiration in LTx recipients both with and without BOS has been investigated using 24-h impedance- $\mathrm{pH}$ recordings and detection of pepsin and bile acids in BALF. The main findings were as follows: 1) $49 \%$ of the patients had increased GOR; 2) LTx patients with CF had the highest prevalence of GOR; 3) gastric aspiration occurred frequently after LTx, as shown by the presence of pepsin in BALF of all patients and bile acids in BALF of $50 \%$ of patients; 4) patients with BOS did not have increased GOR and did not have a particularly higher concentration of pepsin in BALF; 5) $70 \%$ of BOS patients had detectable bile in their BALF compared with $31 \%$ of stable LTx patients; and 6) nonacid reflux and degree of gastric aspiration was not lower in patients taking PPI treatment.

Using oesophageal impedance-pH monitoring, the most sensitive method available for reflux detection [19], less GOR was found over $24 \mathrm{~h}$ in LTx patients than the previously reported prevalence of $69.8-78 \%$ [7, 12, 20]. The selection criteria for recruitment in the present study were different: in the present study, patients were chronologically recruited for impedance-pH measurement, regardless of the presence of reflux symptoms, while in some other studies, at least a subset of patients was recruited on the basis of reflux symptoms and data were collected retrospectively $[7,12]$. The later time after LTx of recording impedance-pH may also explain the lower prevalence of reflux observed in the present population. In the present study, the patients were monitored for reflux much later than in previous reports $[7,12,20]$. Reflux rates might be higher early after transplantation due to surgery-induced anatomical changes that improve with time. This hypothesis requires experimental confirmation.

In the LTX patients with abnormal reflux indices, a small number of LTX patients was diagnosed with exclusively increased nonacid reflux. This population comprised 27 and
$71 \%$ of the LTX patients with GOR, studied off PPI and on PPI, respectively, and would not have been diagnosed with $\mathrm{pH}$ monitoring alone.

It was confirmed that CF patients have an increased prevalence of acid reflux [21]. In addition, it has been shown that CF patients not only have acid but also nonacid reflux and, more often, proximal reflux when compared with other LTx recipients. They also had a trend towards higher pepsin levels in BALF and significantly more CF patients had bile acids in BALF, confirming that they are prone to gastric aspiration.

The present study is the first to compare both markers of gastric aspiration in the same patients. All LTx recipients had increased levels of pepsin in BALF, suggesting that aspiration after LTx might be a ubiquitous event, even in those patients with normal GOR indices. These data confirm earlier findings $[9,22]$. Bile acids have also been detected in BALF in $49 \%$ of LTx patients and none of the nontransplant samples, which was similar to the results reported by D'OvIDIO and coworkers $[8,10]$. The slightly lower absolute levels of bile acids in BALF in the present study may be due to a different BALF procedure and assay.

The current data thus suggest that the presence of pepsin or bile acids in BALF provides different information: pepsin is a general marker of aspiration of gastric content, while bile acids are a more specific marker of gastric aspiration, which might be specifically related to the pathophysiology of aspirationinduced BOS.

In the current cross-sectional study, an increased prevalence of reflux (acid or nonacid) in patients with BOS compared with stable LTx recipients could not be found, nor could a significant correlation between reflux and FEV1. A few crosssectional studies have previously looked at the effect of reflux on the pulmonary function and the development of BOS after LTx. DAVIS et al. [12] described an equally increased oesophageal acid exposure in patients both with and without BOS. HADJILIADIs et al. [7] described no difference in the prevalence of abnormal oesophageal $\mathrm{pH}$ results between patients with or without allograft dysfunction, although a significant negative correlation was found between acid exposure and FEV1. In a prospective study in 48 patients, it was shown that abnormal $\mathrm{pH}$ testing at 3 months after LTx was associated with a significantly reduced time to development of BOS [10]. These results are different from the current data and might be affected by the timing of the reflux testing and the type of diagnostic procedure. In most studies, $\mathrm{pH}$ testing without impedance was performed 3-6 months after LTx, while in the current study, impedance $\mathrm{pH}$ was performed $>1$ yr after LTx. Conversely, in the study by D'OvidIO et al. [10], a slightly higher prevalence of abnormal $\mathrm{pH}$ testing was found at 12 months than at 3 months after LTx. It was not reported if abnormal $\mathrm{pH}$ testing at 12 months was also associated with a reduced freedom from BOS.

In the present study, an increased concentration of pepsin was not found in BALF of patients with BOS compared with stable LTx patients. Conversely, an increased presence of bile acids was found in patients with BOS $\geqslant 1$ when compared with patients without BOS. These data are consistent with the data by D'OviDio et al. [8], who also demonstrated an association 
between the presence of bile acids in BALF and the onset of BOS. The present results confirm that in addition to testing for GOR, detection of the presence of bile acid aspiration might also be worthwhile in order to determine those LTx patients that are prone to GOR-induced BOS.

Previous studies suggested that LTx recipients with GOR may benefit from anti-reflux treatment (either pharmacologically or surgically) $[11-13,23,24]$. It is a common practice after LTx to prescribe prophylactic therapy with PPI. The present results from the separate group of patients studied while on PPI showed that treatment significantly reduced oesophageal acid exposure and the number of acid reflux events, although patients still had nonacid GOR. Moreover, the levels of pepsin in BALF and the proportion of patients with detectable bile acids were similar in patients on PPI compared with patients off PPI. This suggests that PPI treatment, effectively reducing gastric acid secretion, does not prevent gastric aspiration and it is probable that it will not protect against a GOR-induced impairment of lung allograft.

The current authors hypothesise that aspiration of gastric content in LTx patients might occur, not only because of increased GOR but also in the context of normal or even a reduced number of reflux events (acid and nonacid). The contribution of reflux to the development of BOS might be more related to a particular component of the refluxate rather than its frequency or volume. This is supported by the finding of significant improvement of lung function and freedom from BOS occurring after surgical fundoplication, the most radical anti-reflux procedure [11, 12, 23]. Survival after early fundoplication was significantly better in the present study than in those who had no evidence of acid GOR, defined as having a normal $\mathrm{pH}$ monitoring [11]. A possible explanation for this paradoxical observation might thus be that a number of patients with normal $\mathrm{pH}$ monitoring results might still be experiencing subclinical aspiration, triggering the development of BOS.

The main limitation of the present study is its cross-sectional design. The main advantages are the use of impedance $\mathrm{pH}$ and the combined measurement of two markers of aspiration in BALF. Further prospective studies using the same reflux indices and aspiration markers are needed to evaluate the effect of reflux on the long-term evolution of the lung function and the development of BOS. This should also allow refinement of the specific indications for anti-reflux surgery in LTx recipients.

In conclusion, the current authors found that by using a state-ofthe-art diagnostic procedure, one-half of the lung transplant patients had increased gastro-oesophageal reflux, in part nonacid gastro-oesophageal reflux. Patients with bronchiolitis obliterans syndrome did not have particularly higher reflux indices. Gastric aspiration occurs frequently in lung transplantation patients, as shown by the presence of pepsin in bronchoalveolar lung fluid of all patients and bile acids in bronchoalveolar lung fluid of $50 \%$ of the patients. Bronchoalveolar lung fluid pepsin is a general marker of aspiration of gastric content, whereas bile acid in bronchoalveolar lung fluid is more specific and might be associated with the development of bronchiolitis obliterans syndrome. Treating patients with proton pump inhibitor does not protect patients from aspiration of gastric contents and probably will not prevent the development of bronchiolitis obliterans syndrome.

\section{APPENDIX \\ Methodology of pH-impedance measurement}

Oesophageal impedance-pH was recorded with a $2.1 \mathrm{~mm}$ diameter catheter that comprised six electrode pairs to measure intraluminal impedance and two antimony $\mathrm{pH}$ sensors (Sandhill Scientific, Inc., Highlands Ranch, CO, USA). The catheter was passed transnasally and positioned to record $\mathrm{pH}$ in the stomach, and $\mathrm{pH}$ and impedance in the oesophageal body. Oesophageal $\mathrm{pH}$ was measured at $5 \mathrm{~cm}$ and impedance at $3,5,7,9,15$ and $17 \mathrm{~cm}$ proximal to the lower oesophageal sphincter (LOS; see figure in Appendix). The impedance-pH catheter was connected to an ambulatory device containing the amplifiers (Sleuth; Sandhill Scientific, Inc.). The impedance amplifier delivered ultra-low current in a range of $1-2 \mathrm{kHz}$, with resulting current flow variations in response to intraluminal impedance changes. The impedance and $\mathrm{pH}$ signals were digitised at $50 \mathrm{~Hz}$ and stored in the data logger. Before the start of the recording, the $\mathrm{pH}$ electrodes were calibrated using $\mathrm{pH} 4.0$ and $\mathrm{pH} 7.0$ buffer solutions. Patients were asked to remain upright during the day and only lie down at their usual bedtime. Event markers on the data logger recorded meal times and posture changes. Between meals, patients abstained from snacks, beverages with a $\mathrm{pH}<5$, and were asked to avoid lozenges and chewing gum. Before the study, patients were instructed to keep a careful diary and were trained to use a dedicated event marker in the data logger, to record cough episodes and other events.

Total 24-h oesophageal acid exposure was calculated as the percentage of time that the oesophageal $\mathrm{pH}$ was $<4$ and was

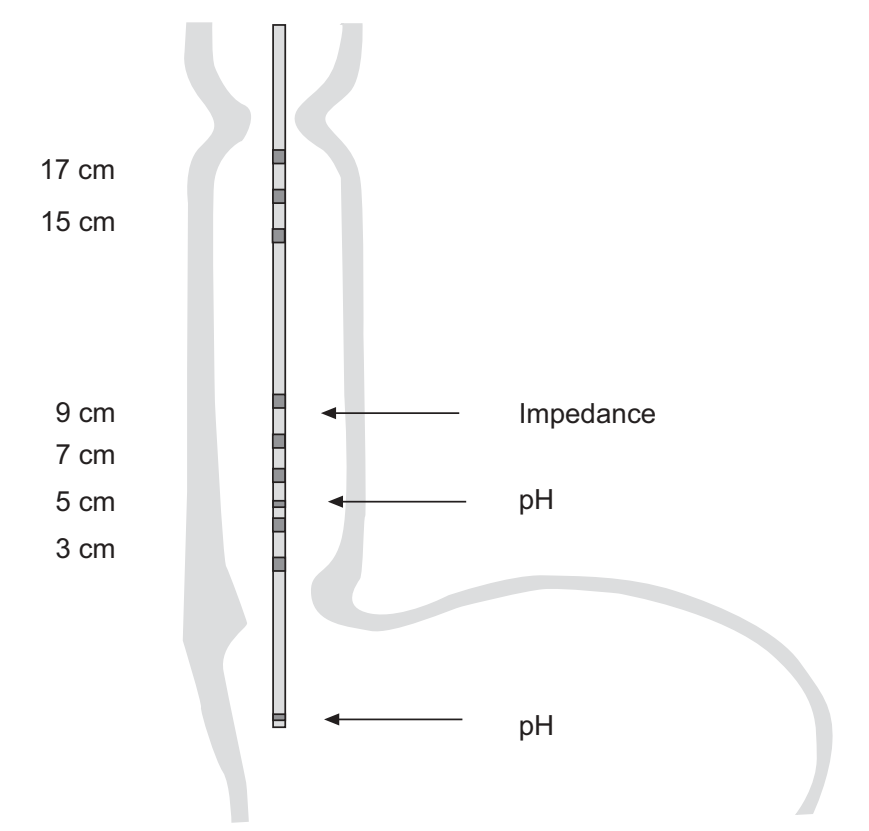

FIGURE Schematic representation of the impedance-pH catheter, positioned in the oesophagus. The proximal $\mathrm{pH}$ electrode is positioned $5 \mathrm{~cm}$ above the lower oesophageal sphincter and the distal electrode in the stomach, allowing impedance measurements at 3, 5, 7, 9, 15 and $17 \mathrm{~cm}$ above the lower oesophageal sphincter. 
considered increased if $\mathrm{pH}$ was $<4$ for $>4.5 \%$ of the recording time.

Individual reflux events, detected by impedance, were counted and classified according to the corresponding $\mathrm{pH}$ change as acid and nonacid. A GOR event was defined as a sequential orally progressing drop in impedance to $<50 \%$ of the baseline values starting distally ( $3 \mathrm{~cm}$ above the LOS) and propagating retrograde to at least the next two more proximal measuring segments. Reflux was classified as acid if the $\mathrm{pH}$ fell to $<4$ for $\geqslant 4$ s or, if $\mathrm{pH}$ was already $<4$, as a decrease of $\geqslant 1 \mathrm{pH}$ unit sustained for $>4 \mathrm{~s}$. Nonacid reflux was defined as a $\mathrm{pH}$ drop of $\geqslant 1 \mathrm{pH}$ unit sustained for $>4 \mathrm{~s}$ with the basal $\mathrm{pH}$ remaining between 4 and 7 . The number of reflux events was regarded as increased if $>95$ th percentile of normal data obtained in healthy subjects [17].

For each reflux episode detected by impedance, the volume exposure at $5 \mathrm{~cm}$ above LOS was calculated as the time (s) between the $50 \%$ drop in impedance until the $50 \%$ recovery of the impedance baseline. Total volume exposure $24 \mathrm{~h}^{-1}$ was obtained by addition of volume exposure of all individual reflux events. The volume exposure was regarded as increased if above the 95th percentile of data obtained in healthy subjects [17].

The proximal extent of reflux was evaluated from the impedance tracings and expressed as total number of reflux episodes reaching $15 \mathrm{~cm}$ above the LOS.

The gastric acid exposure was measured and was used to evaluate the effect of proton pump inhibitors on gastric acid secretion. The effect of the medication was considered adequate if the gastric $\mathrm{pH}$ was acid $(\mathrm{pH}<4)$ during $<50 \%$ of the time.

\section{REFERENCES}

1 Trulock EP, Edwards LB, Taylor DO, et al. Registry of the International Society for Heart and Lung Transplantation: twenty-third official adult lung and heart-lung transplantation report - 2006. J Heart Lung Transplant 2006; 25: 880-892.

2 Verleden GM, Dupont LJ, Van Raemdonck DE. Is it bronchiolitis obliterans syndrome or is it chronic rejection: a reappraisal? Eur Respir J 2005; 25: 221-224.

3 Boehler A, Kesten S, Weder W, et al. Bronchiolitis obliterans after lung transplantation: a review. Chest 1998; 114: 1411-1426.

4 Verleden GM, Bankier A, Boehler A, Corris P, Dupont LJ. Bronchiolitis obliterans syndrome after lung transplantation: diagnosis and treatment. Eur Respir Mon 2004; 29: 1-25.

5 Verleden GM, Dupont LJ. Obliterative bronchiolitis: incidence, clinical features, histology, diagnosis, and treatment. In: Lynch JP, Ross D, eds. Lung and HeartLung Transplantation. Lung Health and Biology in Disease Series. Volume 217. New York, Marcel Dekker, Inc., 2006.

6 Benden C, Aurora P, Curry J, et al. High prevalence of gastroesophageal reflux in children after lung transplantation. Pediatr Pulmonol 2005; 40: 68-71.

7 Hadjiliadis D, Duane Davis R, Steele MP, et al. Gastroesophageal reflux disease in lung transplant recipients. Clin Transplant 2003; 17: 363-368.
8 D'Ovidio F, Mura M, Tsang M, et al. Bile acid aspiration and the development of bronchiolitis obliterans after lung transplantation. J Thorac Cardiovasc Surg 2005; 129: 1144-1152.

9 Ward C, Forrest IA, Brownlee IA, et al. Pepsin like activity in bronchoalveolar lavage fluid is suggestive of gastric aspiration in lung allografts. Thorax 2005; 60: 872-874.

10 D'Ovidio F, Mura M, Ridsdale R, et al. The effect of reflux and bile acid aspiration on the lung allograft and its surfactant and innate immunity molecules SP-A and SP-D. Am J Transplant 2006; 6: 1930-1938.

11 Cantu E, Appel JZ, Hartwig MG, et al. Early fundoplication prevents chronic allograft dysfunction in patients with gastroesophageal reflux disease. Ann Thorac Surg 2004; 78: 1142-1151.

12 Davis RD, Lau CL, Eubanks S, et al. Improved lung allograft function after fundoplication in patients with gastroesophageal reflux disease undergoing lung transplantation. J Thorac Cardiovasc Surg 2003; 125: 533-542.

13 Palmer SM, Miralles AP, Howell DN, et al. Gastroesophageal reflux as a reversible cause of allograft dysfunction after lung transplantation. Chest 2000; 118: 1214-1217.

14 Mainie I, Tutuian R, Shay S, et al. Acid and non-acid reflux in patients with persistent symptoms despite acid suppressive therapy: a multicentre study using combined ambulatory impedance - $\mathrm{pH}$ monitoring. Gut 2006; 55: 1398-1402.

15 Estenne M, Maurer JR, Boehler A, et al. Bronchiolitis obliterans syndrome 2001: an update of the diagnostic criteria. J Heart Lung Transplant 2002; 21: 297-310.

16 Sifrim D, Castell D, Dent J, et al. Gastro-oesophageal reflux monitoring: review and consensus report on detection and definitions of acid, non-acid, and gas reflux. Gut 2004; 53: 1024-1031.

17 Sifrim D, Blondeau K. Technology insight: the role of impedance testing for esophageal disorders. Nat Clin Pract Gastroenterol Hepatol 2006; 3: 210-219.

18 Young LR, Hadjiliadis D, Davis RD, et al. Lung transplantation exacerbates gastroesophageal reflux disease. Chest 2003; 124: 1689-1693.

19 Button BM, Roberts S, Kotsimbos TC, et al. Gastroesophageal reflux (symptomatic and silent): a potentially significant problem in patients with cystic fibrosis before and after lung transplantation. J Heart Lung Transplant 2005; 24: 1522-1529.

20 Stovold R, Forrest IA, Corris PA, et al. Pepsin, a biomarker of aspiration in lung allografts: a putative association with rejection. Am J Respir Crit Care Med 2007; 175: 1298-1303.

21 Lau CL, Palmer SM, Howell DN, et al. Laparoscopic antireflux surgery in the lung transplant population. Surg Endosc 2002; 16: 1674-1678.

22 O'Halloran EK, Reynolds JD, Lau CL, et al. Laparoscopic Nissen fundoplication for treating reflux in lung transplant recipients. J Gastrointest Surg 2004; 8: 132-137.

23 Zerbib F, des Varannes SB, Roman S, et al. Normal values and day-to-day variability of 24-h ambulatory oesophageal impedance $-\mathrm{pH}$ monitoring in a Belgian-French cohort of healthy subjects. Aliment Pharmacol Ther 2005; 22: 1011-1021.

24 Nicod LP. Mechanisms of airway obliteration after lung transplantation. Proc Am Thorac Soc 2006; 3: 444-449. 